\title{
Non-adiabatic passing electron response and outward impurity convection in gyrokinetic calculations of impurity transport in ASDEX Upgrade plasmas
}

\author{
C. Angioni ${ }^{1}$, R. Dux ${ }^{1}$, E. Fable ${ }^{2}$, A.G. Peeters ${ }^{3}$ and the \\ ASDEX Upgrade Team \\ 1 Max-Planck Institut für Plasmaphysik, IPP-Euratom Association, D-85748 \\ Garching bei München, Germany \\ ${ }^{2}$ Centre de Recherches en Physique des Plasmas, Association \\ Euratom-Confédération Suisse, EPFL, 1015 Lausanne, Switzerland \\ 3 University of Warwick, CV4 7AL, Coventry, United Kingdom
}

\begin{abstract}
Gyrokinetic calculations with the GS2 code of impurity transport in ASDEX Upgrade $\mathrm{H}$-mode plasmas are presented. A method to separate the diagonal and off-diagonal terms in the quasi-linear flux computed by the gyrokinetic code for a trace impurity is introduced and applied. It is shown that in the experimental conditions of strong central electron cyclotron heating, unstable modes rotating in the electron diamagnetic direction are excited in the central region of the plasma. These are generated by the non-adiabatic response of passing electrons and feature extremely elongated eigenfunctions along the field line. The related fluctuations in the electrostatic potential generate an outward convection of the impurities. This theoretical findings are in agreement with the outward convection of laser ablated Si measured in these experimental conditions. In contrast, in the outer region of the plasma, as well as everywhere in the case of discharges without central electron heating, ion temperature gradient modes produce an impurity pinch directed inwards, which is also in agreement with the experimental observations.
\end{abstract}




\section{Introduction and motivations}

Central impurity accumulation may imply a severe limitation for operation and performance in a fusion reactor. Therefore, the possibility of reaching plasma conditions in which the convection of highly charged impurities in the core region is directed outwards is extremely attractive for reactor operation, particularly if a radiative belt of impurities has to be produced in the edge in order to reduce the heat loads on the walls. Recent experimental studies have identified discharge conditions in which the impurity convection, measured in laser blow-off experiments, is small or even directed outwards $[1,2,3]$. The experimental results indicate that a necessary, although perhaps not sufficient, ingredient to obtain such an experimental behaviour of the impurity convection is central electron heating. This raises questions about the effect of $\alpha$ particle heating on impurity transport in a burning plasma, and about the possibilities of controlling impurity accumulation in a reactor by external means.

From the theoretical standpoint, the identification of physics mechanisms by which impurities can be convected outwards, and the assessment of the parameter domain in which such mechanisms are expected to take place, provides an important set of predictions which can be tested in present experiments. Such a theoretical investigation and related comparison with present experiments is also the way to be undertaken to gain the capability of predicting the impurity behaviour in a burning plasma.

More specifically, within the theory of core plasma microinstabilities, it has been pointed out recently that the parallel compression of fluctuations along the field line of the parallel velocity of the impurity can generate a radial convection which is directed outwards for modes propagating in the electron diamagnetic direction [4].

In the present paper, we investigate whether this mechanism is able to produce a total impurity convection directed outwards in experimental conditions in which an outward convection was measured and we analyse the physics processes by which it takes place.

To this purpose, we compute the quasi-linear impurity transport with the gyrokinetic code GS2 [8, 9] for the plasma parameters measured in specific ASDEX Upgrade (AUG) discharges in which Si laser ablations were carried out expressely to measure pinch and diffusivity of the impurity [1]. We show that such a theoretical investigation on experimentally measured data leads to the discovery of an interesting physical mechanism which couples the dynamics of passing electrons with the parallel dynamics of the impurities, through the resulting fluctuations of the electrostatic potential. Such an interaction between the non-adiabatic passing electron response and the parallel dynamics of the impurities is presented here for the first time. A radial impurity convection directed outwards is produced in the presence of these impurity parallel velocity fluctuations, along the physics process described in Ref. [4].

In the next section the experimental results are reviewed, giving particular emphasis to the parameters which are relevant for the destabilisation of core plasma microinstabilities, namely ion temperature gradient (ITG), trapped electron modes 
(TEM) and electron temperature gradient (ETG) modes, and which are used in input of the gyrokinetic calculations. Section 3 shows the results of gyrokinetic calculations performed to identify the most unstable modes predicted to take place in these plasmas at different radial positions. Section 4 describes the method applied to separate the different contributions to the impurity flux in a diagonal part, namely proportional to the density gradient, and in residual convective parts, both off-diagonal, namely proportional to the impurity temperature gradient, and pure convection. In Section 5, the impurity transport produced by the unstable modes is computed and the related physics processes are investigated, focusing on conditions which lead to an outward total impurity convection. In Section 6, the parameter domain is defined in which a total outward convection is found in the numerical results, by varying various plasma parameters. Finally in Section 7, conclusions of this work are drawn.

\section{Experimental results}

A systematic experimental study of the effects of different heating profiles on the transport of a laser ablated Si impurity was performed in AUG during the 2002 and 2003 experimental campaigns [1]. In a series of type-I ELMy $\mathrm{H}$-mode discharges producing plasmas at $0.8 \mathrm{MA}$, with line average densities around $5.510^{19} \mathrm{~m}^{-3}$, heated by $5 \mathrm{MW}$ of neutral beam injection (NBI), the addiction of $0.8 \mathrm{MW}$ of strongly localized central electron cyclotron heating $(\mathrm{ECH})$ was observed to increase the central Si diffusion coefficient by one order of magnitude with respect to the phase with NBI heating only. The measured ratio $V / D$ of the impurity convection to the impurity diffusivity in the plasma centre was also strongly modified, from a large negative (directed inwards) value of $V / D$ during the NBI only phase, to a small positive (directed outwards) value of $V / D$ during the central ECH phase. The profiles of the electron and ion temperatures, as well as of the electron density obtained during the two heating phases are plotted in Fig. 1(a), 1(b) and 1(c) respectively. The electron temperature profile is measured by electron cylotron emission radiometry, the ion temperature profile by charge exchange recombination spectroscopy, while the electron density profile by a Thomson scattering diagnostics. The measurements have been averaged over stationary time windows of 0.5 $\mathrm{s}$ during the two heating phases. The normalized minor radius on which the profiles are plotted is defined by the half width of the corresponding flux surface. This choice is made since this is the definition of minor radius which is later used in the GS2 gyrokinetic calculations, and will be referred as $\hat{r}_{G S 2}=r_{G S 2} / a_{G S 2}$, where $a_{G S 2}$ is the half width of the last closed flux surface.

We observe that central ECH, absorbed around $\hat{r}_{G S 2}=0.1$, produces a strong steepening of the electron temperature profile in the very central region of the plasma column. Concurrently, the ion temperature profile flattens slightly in the central region, while the density profile peaks slightly over almost the full minor radius. Such a behaviour of the density profile is opposite to the usual observations in low density plasmas, where the density profile flattens in response to central ECH. The difference 
in these density behaviours in AUG was investigated in previous works [10, 11].

The variations of the profile shapes are more precisely quantified in Fig. 1(d),1(e) and $1(\mathrm{f})$, where the corresponding normalized logarithmic temperature and density gradients $R / L_{T i}, R / L_{T e}$ and $R / L_{n}$ are plotted. These parameters are those used in input of the gyrokinetic code, and consistently all the radial gradients have been computed with respect to $r_{G S 2}$, while $R$ is the geometrical major radius. Shaded areas in the plots are those over which average values of the logarithmic gradients have been considered in the gyrokinetic calculations, centered at $\hat{r}_{G S 2}=0.2$ and $\hat{r}_{G S 2}=0.5$.

\section{Unstable modes}

In this section, the first step of the theoretical analysis of the AUG plasmas described in the previous section is presented. It is dedicated to the identification of the most unstable modes at two radial locations, corresponding to the shaded regions shown in Fig.1, and it is performed by gyrokinetic calculations with the code GS2 using in input experimentally measured plasma parameters. The calculations are performed including the effects of geometry by means of a local description of the equilibrium proposed by Miller [5]. The equilibrium reconstruction has been performed with the CLISTE code $[6,7]$, regularly used for AUG plasmas. The most important input parameters used are listed in Table 1. The spectra of the most unstable modes found in these calculations are presented in Fig. 2. The growth rate $\gamma$, the real frequency $\omega_{r}$ of the most unstable mode as well as the ratio $\gamma /\left\langle k_{\perp}^{2} \rho_{i}^{2}\right\rangle$ are plotted as a function of $k_{\theta} \rho_{i}$. The ratio $\gamma /\left\langle k_{\perp}^{2} \rho_{i}^{2}\right\rangle$ is interesting because, as noted in [14], it allows, on the basis of linear gyrokinetic calculations, an estimate of the scale, namely the value of $k_{\theta} \rho_{i}$, at which the transport is likely to be maximum in the nonlinear saturated state. As noted in several works $[14,15,16]$, particle transport in nonlinear saturated states is provided almost exclusively by the long scales (small $k_{\theta} \rho_{i}$ range) and not by the scales at which linear growth rates are usually at their maximum. Moreover, particle fluxes can even change sign as a function of $k_{\theta} \rho_{i}$. Therefore, according to the quasi-linear model proposed in $[14,16]$, we compute the quasi-linear fluxes in the range of $k_{\theta} \rho_{i}$ which is the one producing the transport in the nonlinear state, and not in the range in which the largest linear growth rate is located.

Fig. $2(\mathrm{a}-\mathrm{c})$ shows the spectra for the plasma with NBI heating and central ECH at $\hat{r}_{G S 2}=0.2$, Fig. $2(\mathrm{~d}-\mathrm{f})$ shows the spectra for the plasma with NBI heating and without central ECH, again at $\hat{r}_{G S 2}=0.2$, while Fig. $2(\mathrm{~g}-\mathrm{h})$ shows the spectra for the plasma with NBI and central ECH at $\hat{r}_{G S 2}=0.5$. The case at $\hat{r}_{G S 2}=0.5$ without ECH is not showns, since it is very similar to the latter, as one can deduce also from the very little change in plasma parameters presented in Fig. 1.

We observe that in the central part of the plasma $\left(\hat{r}_{G S 2}=0.2\right)$ the addition of ECH implies a reversal of the direction of propagation of the most unstable mode from the ion direction (positive sign of $\omega_{r}$ in Fig 2(e) ) to the electron direction (negative sign of $\omega_{r}$ in Fig 2(b) ). We observe also that no local maximum of $\gamma$ as a function of $k_{\theta} \rho_{i}$ is 
found in this case in the usual ITG/TEM range. A maximum is found only in the ETG range, although an unstable mode is already present in the low-k range. We mention that, however, in the high-k range the particle transport of such a mode becomes small, as indicated by the behaviour of the ratio $\gamma /\left\langle k_{\perp}^{2} \rho_{i}^{2}\right\rangle$, and as more consistently shown by fully nonlinear simulations $[15]$.

At $\hat{r}_{G S 2}=0.5$ the usual ITG mode is found, with maximum growth rate around $k_{\theta} \rho_{i}=0.4$, but peak of $\gamma /\left\langle k_{\perp}^{2} \rho_{i}^{2}\right\rangle$ around $k_{\theta} \rho_{i}=0.15$, completely consistent with nonlinear spectra of ITG transport (see e.g. [12]).

An ITG is still found as dominant mode in the more central case, when central ECH is absent. The peak of $\gamma /\left\langle k_{\perp}^{2} \rho_{i}^{2}\right\rangle$ is found around 0.3 , due to the presence of modes strongly elongated along the field line found at the lowest $k_{\theta} \rho_{i}$ values, allowed by the small value of the magnetic shear in this plasma region.

The mode rotating in the electron diamagnetic direction found in the low-k range in the case around $\hat{r}_{G S 2}=0.2$ with ECH is not a TEM. This might have been expected from the rather small value of the local inverse aspect ratio $\epsilon=r_{G S 2} / R_{\text {geo }}=0.068$. However, the magnetic shear is small and the value of $R / L_{T e}$ is rather large. In fact, an estimate of the threshold from [13] yields a value of 6 for these parameters, i.e. below the experimental value. Analysis has shown that collisions together with the small value of $\epsilon$ lead to a large effective collision frequency $\nu_{e i} / \epsilon$ (an effect not kept in the formula of [13]), which reduces significantly the growth rate of the TEM. To identify the nature of this mode, a more detailed investigation is required. To this purpose, in Fig. 3(a-b) we plot the eigenfunction of the mode $\left|\Phi^{2}\right|$ as well as the related phase relationship between electron density fluctuations $\tilde{n}_{e}$ and electrostatic potential fluctuations $\Phi$, namely $\operatorname{Im}\left(\tilde{n}_{e} \Phi^{*}\right) /\left\langle\left|\Phi^{2}\right|\right\rangle$, as a function of the field line, more precisely of the ballooning coordinate $\theta$, for the value of $k_{\theta} \rho_{i}=0.24$, namely at the value where $\gamma /\left\langle k_{\perp}^{2} \rho_{i}^{2}\right\rangle$ peaks. In the previous expression, the apex $*$ indicates the complex conjugate, while the symbol \langle\rangle indicates flux surface average. We recall that the quasi-linear radial particle flux of the species $j$ can be computed by $\Gamma_{j}=\operatorname{Re}\left\langle\tilde{n}_{j} \tilde{v}_{E \times B}\right\rangle=k_{\theta} \operatorname{Im}\left\langle\tilde{n}_{j} \Phi^{*}\right\rangle$, and therefore it is directly proportional to the above defined phase relationship.

For comparison, the same quantities for the corresponding ITG case obtained at $\hat{r}_{G S 2}=0.2$ for the plasma without ECH are plotted in Fig.3 (c-d).

We observe that the eigenfunction obtained in the ECH case is extremely elongated along the field line and is mainly slab in nature. Indeed a window over the ballooning angle $\theta$ covering 39 turns in the toroidal direction has been necessary in the calculation to fully resolve it. For comparison, the ITG mode found in the case without ECH is much more localized around $\theta=0$, despite the same low value of magnetic shear $s=0.2$. Such a behaviour, namely the formation of these large envelope of the eigenfunction along the field line, is generated by the non-adiabatic response of the passing electrons, and was already observed in the literature [17].

As it can be seen from Fig. 3(b), passing electrons provide a particle flux, which is directly proportional to the phase relationship $\operatorname{Im}\left(\tilde{n}_{e} \Phi^{*}\right) /\left\langle\left|\Phi^{2}\right|\right\rangle$, which is directed inwards (negative sign in the figure). This is also visible in Fig. 4, in which the 
same phase relationship has been plotted as a function of the pitch angle parameter $\lambda=\mu B_{0} / E$, with the magnetic moment $\mu=m_{e} v_{\perp}^{2} /(2 B)$ and the kinetic energy $E=m_{e} v^{2} / 2$. Again, for comparison, the dashed line shows the same quantity for the NBI case. While the latter shows the typical behaviour of a collisional ITG case, with trapped electrons moving outwards (positive sign in the figure) and passing being practically adiabatic, namely without any strong phase shift from the electrostatic potential, the ECH case shows a strong phase shift of the passing electrons from the electrostatic potential, with passing electrons convecting inwards. The present results, and in particular the inward pinch brought by non-adiabatic passing electrons, are completely analogous to those described in Ref. [17]. Interestingly, we show here that they take place in the gyrokinetic simulation of some experimentally measured ASDEX Upgrade conditions. More important within the purpose of the present study, we show in the next sections that when these kind of modes are destabilized, an effect on the transport of impurities is produced, with the eventual consequence of producing an outward convection of impurities, as it was measured in these conditions.

\section{Computation of quasi-linear trace impurity transport with GS2}

In order to compute the transport of an impurity present in the plasma at very low concentration we include a third particle species in the gyrokinetic calculations. The gyrokinetic equation for a particle species is linear in the logarithmic gradients of the temperature and density of that particle species. As a direct consequence, the linear response of the density perturbation $\tilde{n}_{j}$ of a particle species identified by the index $j$ can be expressed formally by a linear combination of the logarithmic gradients in the form

$$
\tilde{n}_{j}=\left(A_{j} \frac{R}{L_{n j}}+B_{j} \frac{R}{L_{T j}}+C_{j}\right) \phi .
$$

The complex coefficients $A_{j}, B_{j}$ and $C_{j}$ are complicated functions of the linear eigenvalues. The quasi-linear particle flux $\Gamma_{j}$, provided by the product $\left\langle\tilde{n}_{j} \tilde{v}_{E \times B}\right\rangle$ is proportional to $\operatorname{Im}\left(\tilde{n}_{j} \phi^{*}\right)$ and therefore preserves the linearity in the logarithmic gradients

$$
\Gamma_{j} \propto\left[\operatorname{Im}\left(A_{j}\right) \frac{R}{L_{n j}}+\operatorname{Im}\left(B_{j}\right) \frac{R}{L_{T j}}+\operatorname{Im}\left(C_{j}\right)\right]\left|\phi^{2}\right|
$$

which can be recasted in the more familiar form

$$
\frac{R \Gamma_{j}}{n_{j}}=D \frac{R}{L_{n j}}+D_{T} \frac{R}{L_{T j}}+R V_{P}
$$

where $D$ is the diagonal diffusion coefficient, $D_{T}$ is the (off-diagonal) thermodiffusion coefficient, and $V_{P}$ is the pure convection velocity. In such a description, we define pure convection the residual particle flux obtained when both the (logarithmic) density and temperature gradients are equal to zero. The same general expression can be derived also within a fluid treatment, for which, in some limits, useful analytical expressions for the different coefficients can be derived [18, 19, 4, 20]. 
In general, $A, B$ and $C$, or $D, D_{T}$ and $V_{p}$, depend themselves on $R / L_{n}$ and $R / L_{T}$, through the dependence on the gradients of the eigenvalues, hence this linear dependence is only formal, but actually does not hold in practice, since $A, B$ and $C$ do not remain constant when the gradients are changed.

However, if the particle species $j$ is present with a charge concentration which is small enough that its contribution to the quasi-neutrality condition can be neglected, namely behaves as a trace (or passive) particle, then any modification of the logarithmic gradients of the species $j$ does not modify the dispersion relation nor the resulting eigenvalues, neither the values of the coefficients $A, B$ and $C$. Hence the transport of a trace species is a linear function of the logarithmic gradients of the trace species itself. This observation provides a general method to compute the coefficients $D, D_{T}$ and $V_{p}$ by means of linear gyrokinetic calculations [4, 21]. By including a third particle species at very low concentration and by computing the transport changing alternatively the values of $R / L_{n}$ and $R / L_{T}$ of that species, the values of $C_{T}=D_{T} / D$ and $C_{p}=R V_{P} / D$ can be easily identified, from the slopes of the particle flux as a function of the density and temperature logarithmic gradients, as well as from the value of the flux at zero density and temperature gradients, corresponding to the pure convective flux, as can be easily seen from the following expression

$$
\frac{R \Gamma_{j}}{n_{j}}=D\left(\frac{R}{L_{n j}}+C_{T} \frac{R}{L_{T j}}+C_{P}\right) .
$$

We underline that, as long as a single $k_{\theta} \rho_{s}$ value is considered, following the quasilinear model introduced in [14], the coefficients $C_{T}$ and $C_{P}$ do not depend on the saturation amplitude $\left|\phi^{2}\right|$, and therefore their calculation does not require any mixing length argument, regular weak point of any quasi-linear treatment. The coefficients $C_{T}$ and $C_{P}$ are derived directly from the phase relationship between density and electrostatic potential fluctuations of the unstable mode. Within the applied quasi-linear transport model, the saturation amplitude $\left|\phi^{2}\right|$ appears only in the expression of the diffusion coefficient $D$.

Fig. 5 shows the dependence of the particle flux of a Si impurity with a charge concentration $510^{-4}$ as a function of the Si logarithmic density and temperature gradients, in a plasma made of deuterons and electrons. These calculations have been performed with the gyrokinetic code GS2. We observe that these functional dependences are perfectly linear. We emphasize that the GS2 code does treat the three particle species, D, $\mathrm{e}^{-}$, and $\mathrm{Si}$ in the same way, namely as active species. However, since the charge concentration of $\mathrm{Si}$ is very small, the $\mathrm{Si}$ species actually behaves as a trace (or passive particle). We have verified that in general the modification of the plasma parameters and gradients of a third particle species with a charge concentration which is lower than $1 / 1000$ affects the eigenvalues by less than $1 / 100$. 


\section{Gyrokinetic calculations of impurity transport of the AUG plasmas}

In Table 1 the most important input parameters used for the three experimental cases considered are summarized. For each case, a set of GS2 runs at different values of the impurity charge $Z$, with impurity mass $A=2 Z$, has been performed and the coefficients $C_{P}$ and $C_{T}$ computed at each $Z$ along the procedure described in the previous section. In Fig. 6, the values of $R V / D, C_{P}$ and $C_{T}$ as a function of $\mathrm{Z}$ are plotted for the three experimental cases considered, where $R V / D=C_{P}+C_{T} R / L_{T i}$ provides the total convection to diffusion ratio. These results allow a set of considerations.

- The thermodiffusion coefficient $C_{T}$ has a non-negligible effect only at low Z, as expected from the $1 / Z$ dependence of the curvature and $\nabla B$ drifts.

- The thermodiffusion contribution is directed inwards (negative) for modes rotating in the electron drift direction, and outwards (positive) for modes rotating in the ion drift direction, consistently with analytical fluid models [18, 19, 4, 20], and fluid turbulent simulations [20].

- The convection coefficient $C_{P}$ is usually directed inwards (negative), producing an impurity pinch, but it is directed outwards (positive) in the case at $\hat{r}_{G S 2}=0.2$ with central ECH. The total convection to diffusion ratio $R V / D$ for $\mathrm{Si}(\mathrm{Z}=14, \mathrm{~A}=28)$ is positive (total outward convection) only in that case.

The latter result is in agreement with the experimental observations, namely only in the case at $\hat{r}_{G S 2}=0.2$ with $\mathrm{ECH}$ an outward convection of the laser ablated $\mathrm{Si}$ was measured. This qualitative agreement with the experiment is certainly promising, but limited to very specific conditions, and therefore certainly cannot be considered as providing a full validation of the predictive capabilities of the present gyrokinetic modelling. It remains that it is certainly of interest, and motivates a more detailed theoretical investigation of the physical mechanism by which an outw1ard impurity convection is obtained in these conditions.

To this purpose, first of all it is of interest to look at the spectra of the quasi-linear particle fluxes as a function of $k_{\theta} \rho_{i}$ for the electrons, the main ions (deuterons), and a trace Si impurity, computed for the input parameters of the case at $\hat{r}_{G S 2}=0.2$ with ECH. In Fig. 7 we plot the spectra of the phase relationships $\operatorname{Im}\left\langle\tilde{n}_{j} \Phi^{*}\right\rangle /\left\langle\left|\Phi^{2}\right|\right\rangle$. As recalled above, the quasi-linear particle flux of a species $j$ at a given value of $k_{\theta} \rho_{i}$ is given by $k_{\theta} \operatorname{Im}\left\langle\tilde{n}_{j} \Phi^{*}\right\rangle$. In Fig. $7($ a) we observe that the electron particle flux is directed outwards (positive sign) for small values of $k_{\theta} \rho_{i}$, while it becomes directed inwards (negative sign) for larger values. We remind that, as shown in Fig. 4, at each value of $k_{\theta} \rho_{i}$, the total electron flux is provided by the sum of two contributions of opposite sign, passing electrons with the largest parallel velocities move inwards, while the residual part of the passing and the trapped electrons move outwards. We find, as it could be expected, that the inward contribution of the passing electrons increases with increasing $k_{\theta} \rho_{i}$, leading to a total particle flux directed inwards for sufficiently small wave lengths. In Fig. $7(\mathrm{~b})$ we have plotted the phase relationship $\operatorname{Im}\left\langle\tilde{n}_{Z} \Phi^{*}\right\rangle /\left\langle\left|\Phi^{2}\right|\right\rangle$ for the Si trace 
with zero logarithmic density and temperature gradients. Since both the Si density and temperature are equal to zero in these calculations, the plotted quantity is directly proportional to the Si pure convection $V_{p}$, or to the coefficient $C_{P}$, as defined in Eqs. (3) and (4). We observe that the phase relationship for the trace Si is positive, namely the Si pure convection is directed outwards, at all the values of $k_{\theta} \rho_{i}$, and this regardless of the direction of the electron flux. This shows that the sign of the results obtained in Fig. 6(a) is not specific to the choice of the value of $k_{\theta} \rho_{i}$ we made in the quasi-linear model, and that an outward impurity convection would have been obtained also in the case an average over a spectrum on $k_{\theta}$ was considered, rather than a single value, as assumed in our model.

To investigate the nature of such an impurity outward convection mechanism, in Fig. $8(\mathrm{a}-\mathrm{c})$ we plot the electrostatic potential fluctuation $\left|\phi^{2}\right|$, the Si parallel velocity fluctuations and the phase relationship $\operatorname{Im}\left(\tilde{n} \Phi^{*}\right) /\left\langle\left|\Phi^{2}\right|\right\rangle$, proportional to the Si radial particle flux, as a function of the ballooning variable $\theta$. We observe that, due to the presence of the large envelope of electrostatic potential fluctuations generated by the non-adiabatic reponse of passing electrons, strong parallel velocity fluctuations of the $\mathrm{Si}$ impurity are excited. In correspondence to these parallel velocity fluctuations, a radial particle flux of $\mathrm{Si}$, which is on average directed outwards, is produced. The mechanism of impurity convection proposed in Ref. [4] based on the compression of parallel velocity fluctuations of the impurity is shown to be at play and large enough to provide a total impurity convection directed outwards in these conditions, due to the large envelope of the electrostatic potential fluctuations generated by the non-adiabatic response of the passing electrons. Fig. 6(a) shows also the experimentally interesting case of $\mathrm{W}(\mathrm{A}=$ 184 ) at the typical ionisation stage $46+$ at $3 \mathrm{keV}$ (open symbols). In this case, $C_{P}$ is lower than in the case $Z=46$ and $A=2 Z$, and confirms that the positive value of $C_{P}$ arises mainly from the above mentioned mechanism linked to the parallel dynamics, and therefore increases with increasing $Z / A$ (see Eq. (10) in Ref. [4]).

The present study provides an interesting example of interaction between passing electrons dynamics and impurity parallel dynamics, which were never pointed out before to our knoweldge. To investigate more deeply this interaction and demonstrate the essential role played by passing electrons, we have performed the same gyrokinetic calculations, including a Si trace, considering an $s-\alpha$ equilibrium. While the $s-\alpha$ equilibrium provides a more approximated description of the experimental equilibrium, it provides the flexibility of allowing us to change the trapped particle fraction by changing directly the local inverse aspect ratio $\epsilon$ in input, without affecting the drifts. The two extreme cases $\epsilon=0$, namely no trapped particles, and $\epsilon=1$, namely no passing particles have been considered. In Fig. 9, the phase relationship $\operatorname{Im}\left(\tilde{n} \Phi^{*}\right) /\left\langle\left|\Phi^{2}\right|\right\rangle$ for both the electrons and the Si trace impurity are plotted. The case with $\epsilon=0$ gives results completely equivalent to those obtained in the runs corresponding to the experimental case at $\hat{r}_{G S 2}=0.2$ with central ECH, namely a very elongated mode structure along the field line, with a total outward convection of the impurity (compare to this purpose Fig. 9(a) with Fig. 3(b) for the electron response, and Fig. 9(b) with Fig. 6(c) for the 
Non-adiabatic passing electron response and outward impurity convection in gyrokinetic calculations of in

impurity response). On the contrary, the case $\epsilon=1$ gives completely different results, with a linear mode strucutre strongly localized around $\theta=0$, and a total impurity convection directed inwards. This shows the essential role played by passing electrons in producing the electrostatic potential fluctuations which lead to the generation of an outward convection of the impurities.

\section{Dependence on parameters of the outward impurity convection}

Given the experimental interest for the existence of an outward convection of impurities in the plasma core, in this section we have explored the parameter domain within which the physics mechanism of outward convection described in the previous section can be found to be at place in the gyrokinetic simulations. To this purpose, keeping fixed the charge and mass of the impurity at values of the fully ionized $\mathrm{Si}$, and starting from a reference case which is the experimental one at $\hat{r}_{G S 2}=0.2$ with central ECH (1st line of Table 1), we have varied singularly various plasma parameters and computed in each case the Si transport, namely the thermodiffusion coefficient $C_{T}$ and the convection coefficient $C_{P}$. The results of this exercise allow us as well to confirm and better understand the physics picture of the outward convection mechanism previously presented.

Figures 10 and 11 show $R V / D, C_{P}$ and $C_{T}$, as well as the real frequency $\omega_{r}$ of the mode as a function of different parameters. In Fig. 10(a) the dependence on the normalized logarithmic electron density gradient is shown. We observe that for the first three values of $R / L_{n}$, from 0 to 3 , a positive $C_{P}$ value, namely outward convection, is found. It decreases with increasing $R / L_{n}$, consistently with the corresponding decrease of the absolute value of the mode frequency (see Eq. (10) in Ref. [4]). The value of $C_{P}$ becomes very close to zero for $R / L_{n}=5$, where $\omega_{r}$ has a jump to more negative values. Indeed, for $R / L_{n}=5$, the nature of the mode changes, and from the electron temperature gradient driven mode with non-adiabatic passing electrons obtained at low values of $R / L_{n}$ and featuring the strongly field-line elongated linear mode structure described in the previous section, we move to a usual $R / L_{n}$ driven mode, strongly ballooning around $\theta=0$, for which the outward convection mechanism is not active any more. This result confirms the fact that the outward convection is generated mainly by the large envelope of the electrostatic potential fluctuations.

In Fig. 10(b-c) the dependences on the logarithmic ion and electron temperature gradients are illustrated. From these, it is clear that a mode propagating in the electron drift direction is needed to have an impurity convection directed outwards. Note also the corresponding opposite reversal of the sign of the thermodiffusion coefficient $C_{T}$, consistent with the analytical fluid model results (see e.g. Eq. (7) in Ref. [4]).

Fig. 11(a) shows the dependence on $T_{e} / T_{i}$. $C_{P}$ increases with increasing electron to ion temperature ratio, as correspondingly the absolute value of the mode frequency does, showing the beneficial effect for a positive $C_{P}$, namely for an outward impurity convection, of a large electron to ion temperature ratio.

Fig. 11(b-c) shows that very similar dependences on magnetic shear and local 
safety factor are found. The increase of both these parameters turns out to reduce the passing electrons non-adiabaticity, by tilting the field line elongated structures and by increasing $k_{\|}$. For large values of shear or q the unstable mode generated by the non-adiabatic response of the passing electron and propagating in the electron drift direction is stabilized, and the consequent outward convection of the impurity is lost. An ITG mode, therefore propagating in the ion drift direction, and strongly ballooning around $\theta=0$ is found, with consequent negative value of $C_{P}$, namely inward impurity convection.

All these results reveal that the mechanism of outward impurity convection presented in this work can take place only in the very central region of the plasma,in conventional tokamak scenarios, where the trapped particle fraction, the shear and the safety factor are small. An additional condition is that the electron heating in the central region of the plasma column is strong and localized enough to realize values of $R / L_{T e}$ which exceed locally $R / L_{T i}$ by almost a factor of two.

\section{Conclusions}

Quasi-linear gyrokinetic calculations with the code GS2 of impurity transport in experimentally measured conditions of AUG $\mathrm{H}$-mode plasmas have been presented. A theoretical explanation has been proposed for the reversal of the direction of the central impurity convection from inwards to outwards measured in response of strongly localised central electron heating in Si laser ablation experiments in AUG. It has been shown that in the presence of a strong central peaking of the electron temperature profile, the numerical results predict the destabilization of a mode generated by the non-adiabatic response of the passing electrons in the innermost region of the plasma column $(r / a \simeq 0.2)$. This mode is chararcterized by a very elongated structure along the field line, and the corresponding electrostatic potential fluctuations generate strong fluctuations of the parallel velocity of the impurities, whose compression generates a radial outward convection. In agreement with this theoretical result, an outward convection was measured for laser ablated $\mathrm{Si}$ in these experimental conditions in AUG. On the contrary, in the absence of central electron heating, or around mid-radius in the plasma, such a mode is not found to be unstable, and a usual ITG mode is predicted to occur by the numerical calculations. The latter generates an inward pinch of the impurities, which is also in agreement with the measurements.

By varying several plasma parameters, we have shown that the outward impurity convection mechanism described in this paper is predicted to take place only in the central region of the plasma and cannot explain measurements of outward convection of the impurities obtained around mid-radius, as found in some recent experiments in JET [3].

Finally, the present results document, for the first time to our knowledge, an agreement among the predictions of the direction of the impurity convection obtained with a gyrokinetic code and a set of experimental observations on impurity transport. 
Non-adiabatic passing electron response and outward impurity convection in gyrokinetic calculations of $i$

While this is certainly a promising step in understanding turbulent impurity transport in tokamaks, these results should not be considered as providing a conclusive validation of the present gyrokinetic modelling for general predicting capabilities of impurity transport. Indeed, it must be underlined that such an agreement is limited to very specific experimental conditions. Very different experimental conditions are still to be examined before the gyrokinetic description applied in this work can be considered reliable for general predicting purposes.

\section{Acknowledgments}

The Authors are grateful to W. Dorland and M. Kotschenreuther for providing the code GS2.

\section{References}

[1] Dux R, Neu R, Peeters A G, Pereverzev G, Mück A, Ryter F, Stober J 2003 Plasma Phys. Control. Fusion 451815

[2] Scavino E, Bakos J, Weisen H 2004 Plasma Phys. Control. Fusion 46857

[3] Puiatti M E, Valisa M, Angioni C, Garzotti L, Mantica P, Mattioli M, Carraro L, Coffey I, Sozzi C 2006 Phys. Plasmas 13042501

[4] Angioni C and Peeters A G 2006 Phys. Rev. Lett 96095003

[5] Miller R L, Chu M S, Greene J M, Lin-Liu Y R, and Waltz R E 1998 Phys. Plasmas 5973

[6] Mc Carthy P J 1999 Phys. Plasmas 63554

[7] Schneider W, Mc Carthy P J, Lackner K, Gruber O, Behler K, Martin P, Merkel R 2000 Fusion Engineering and Design 48127

[8] Kotschenreuther M, Rewoldt G, and Tang W M 1995 Comput. Phys. Commun. 88128

[9] Dorland W, Jenko F, Kotschenreuther M, Rogers B N 2000 Phys. Rev. Lett. 855579

[10] Angioni C, Peeters A G, Garbet X, Manini A, Ryter F and ASDEX Upgrade Team 2004 Nucl. Fusion 44827

[11] Angioni C, Peeters A G, Ryter F, Jenko F, Conway G D, Dannert T, Fahrbach U H, Reich M, Suttrop W, Fattorini L 2005 Phys. Plasmas 12040701

[12] Lin Z and Hahm T S 2004 Phys. Plasmas 111099

[13] Peeters A G, Angioni C, Apostoliceanu M, Jenko F, Ryter F 2005 Phys. Plasmas 12022505

[14] Jenko F, Dannert T, and Angioni C 2005 Plasma Phys. Control. Fusion 47 B195

[15] Estrada-Mila C, Candy J, Waltz R E 2005 Phys. Plasmas 12 022305; Erratum 2005 Phys. Plasmas 12049902

[16] Angioni C, Peeters A G, Jenko F, Dannert T 2005 Phys. Plasmas 12112310

[17] Hallatschek K, Dorland W 2005 Phys. Rev. Lett. 95055002

[18] Frojdh M, Liljestrom M, Nordman H, 1992 Nucl. Fusion 32419

[19] Garbet X, Dubuit N, Asp E, Sarazin Y, Bourdelle C, Ghendrih P, Hoang G T 2005 Phys. Plasmas 12082511

[20] Dubuit N, Garbet X, Parisot T, Guirlet R, and Bourdelle C 2007 Phys. Plasmas 14042301

[21] Fable E, Angioni C, Bottino A et al 2006 34th EPS Conference on Plasma Physics, Warsaw, 2006. 
Non-adiabatic passing electron response and outward impurity convection in gyrokinetic calculations of $i$

\begin{tabular}{|l||c|c|c|c|c|c|c|c|c|}
\hline & $R / L_{T i}$ & $R / L_{T e}$ & $R / L_{n e}$ & $T_{e} / T_{i}$ & $\nu_{e i}\left[c_{s} / R\right]$ & $r_{G S 2} / R$ & $q$ & $s$ & $k_{\theta} \rho_{i}$ \\
\hline$\hat{r}_{G S 2}=0.2$ with ECH & 3.5 & 8.0 & 1.5 & 1.13 & 0.10 & 0.068 & 1.20 & 0.20 & 0.23 \\
\hline$\hat{r}_{G S 2}=0.2$ w/o ECH & 5.5 & 6.2 & 1.45 & 0.90 & 0.11 & 0.068 & 1.20 & 0.20 & 0.29 \\
\hline$\hat{r}_{G S 2}=0.5$ with ECH & 4.8 & 5.5 & 2.7 & 1.04 & 0.26 & 0.176 & 1.70 & 0.84 & 0.15 \\
\hline
\end{tabular}

Table 1.

Table 1. Main GS2 input parameters for the three experimental cases considered. 
Figure 1. (Color online) Temperature and density profiles (a-c), as well as related normalized logarithmic gradients (d-f) during stationary phases for the two AUG Hmode plasmas considered, with and without central ECH. The absorption of the ECH power is localized around $\hat{r}_{G S 2}=0.1$.

Figure 2. (Color online) Spectra as a function of $k_{\theta} \rho_{i}$ of the growth rate $\gamma(\mathrm{a}, \mathrm{d}, \mathrm{g})$, the real frequency $\omega_{r}(\mathrm{~b}, \mathrm{e}, \mathrm{h})$ and the ratio $\gamma /\left\langle k_{\perp}^{2} \rho_{i}^{2}\right\rangle(\mathrm{g}, \mathrm{h}, \mathrm{i})$ for the three local cases considered in the gyrokinetic calculations, around $\hat{r}_{G S 2}=0.2$ for the plasmas with (a-c) and without (d-f) central ECH, and around $\hat{r}_{G S 2}=0.5$ for the plasma with ECH $(g-i)$.

Figure 3. (Color online) Linear mode structure of $\left|\phi^{2}\right|$ (a,c) and phase relationship $\operatorname{Im}\left(\tilde{n} \Phi^{*}\right) /\left\langle\left|\Phi^{2}\right|\right\rangle(\mathrm{b}, \mathrm{d})$ as a function of the ballooning angle $\theta$ at $k_{\theta} \rho_{i}=0.24$ for the cases at $\hat{r}_{G S 2}=0.2$, with $(\mathrm{a}, \mathrm{b})$ and without $(\mathrm{c}, \mathrm{d}) \mathrm{ECH}$.

Figure 4. (Color online) Phase relationship $\operatorname{Im}\left(\tilde{n} \Phi^{*}\right) /\left\langle\left|\Phi^{2}\right|\right\rangle$ as a function of the pitch angle parameter $\lambda=\mu B_{0} / E$ at $k_{\theta} \rho_{i}=0.24$ for the cases at $\hat{r}_{G S 2}=0.2$, with (solid line) and without (dashed line) $\mathrm{ECH}$.

Figure 5. (Color online) Trace Si quasi-linear radial flux [a.u.] as a function of the Si normalized logarithmic density gradient $R / L_{N S i}(\mathrm{a})$ and the Si normalized temperature gradient $R / L_{T S i}$ (b) for different values of $R / L_{T S i}$ and $R / L_{n S i}$ respectively.

Figure 6. (Color online) Total convection to diffusion ratio $R V / D$, pure convection coefficient $C_{P}=R V_{P} / D$ and thermodiffusion coefficient $C_{T}=D_{T} / D$ as a function of the trace impurity charge, with impurity mass equal to twice the charge, for the three different cases considered, around $\hat{r}_{G S 2}=0.2$ with (a) and without (b) ECH and around $\hat{r}_{G S 2}=0.5$ with ECH (c). Open symbols in Fig. 6(a) show values of $R V / D$ (open square) and $C_{P}$ (open circle) for the usual ionization stage of $\mathrm{W}$ in a $3 \mathrm{keV}$ plasma $(\mathrm{Z}=46, \mathrm{~A}=184)$.

Figure 7. (Color online) Phase relationship $\operatorname{Im}\left(\tilde{n} \Phi^{*}\right) /\left\langle\left|\Phi^{2}\right|\right\rangle$ for electrons (full circles) and main ions (open squares) $(\mathrm{a})$, and for a $\mathrm{Si}$ trace $(\mathrm{Z}=14, \mathrm{~A}=28)$ with zero density and temperature logarithmic gradients (b), as a function of the dimensionless wave number $k_{\theta} \rho_{i}$, for the case around $\hat{r}_{G S 2}=0.2$ with ECH.

Figure 8. (Color online) Linear mode structure along the field line of the electrostatic potential fluctuations $\left|\phi^{2}\right|$ (a), of the Si parallel velocity fluctuations $\mid \tilde{u}_{\|}$(b) (c) proportional to the radial Si flux, as a function of the ballooning angle $\theta$ at $k_{\theta} \rho_{i}=0.24$, for the case at $\hat{r}_{G S 2}=0.2$ with $\mathrm{ECH}$. 
Figure 9. (Color online) Phase relationship $\operatorname{Im}\left(\tilde{n} \Phi^{*}\right) /\left\langle\left|\Phi^{2}\right|\right\rangle$, proportional to the radial particle flux, for electrons (a,c) and a Si trace (b,d) as a function of the ballooning angle $\theta$, at $k_{\theta} \rho_{i}=0.24$, for the case at $\hat{r}_{G S 2}=0.2$ with ECH, with an $s-\alpha$ equilibrium in the two limit cases local inverse aspect ratio $\epsilon=0(\mathrm{a}, \mathrm{b})$, corresponding to no trapped particles, and $\epsilon=1(\mathrm{c}, \mathrm{d})$, corresponding to no passing particles. Note the different scale on the $\mathrm{x}$-axis in the two cases.

Figure 10. (Color online) Total convection to diffusion ratio $R V / D$, pure convection coefficient $C_{P}=R V_{P} / D$ and thermodiffusion coefficient $C_{T}=D_{T} / D$ and real frequency of the mode (in $\left[c_{s} / R\right]$ units times 10) as a function of the logarithmic electron density gradient $R / L_{n e}$ (a), the logarithmic ion (b) and electron (c) temperature gradients keeping fixed the other input parameters to the values of the case at $\hat{r}_{G S 2}=0.2$ with ECH, at $k_{\theta} \rho_{i}=0.35$.

Figure 11. (Color online) Total convection to diffusion ratio $R V / D$, pure convection coefficient $C_{P}=R V_{P} / D$ and thermodiffusion coefficient $C_{T}=D_{T} / D$ and real frequency of the mode (in $\left[c_{s} / R\right]$ units times 10) as a function of the electron to ion temperature ratio (a), the magnetic shear (b) and the local safety factor (c) keeping fixed the other input parameters to the values of the case at $\hat{r}_{G S 2}=0.2$ with $\mathrm{ECH}$, at $k_{\theta} \rho_{i}=0.35$.
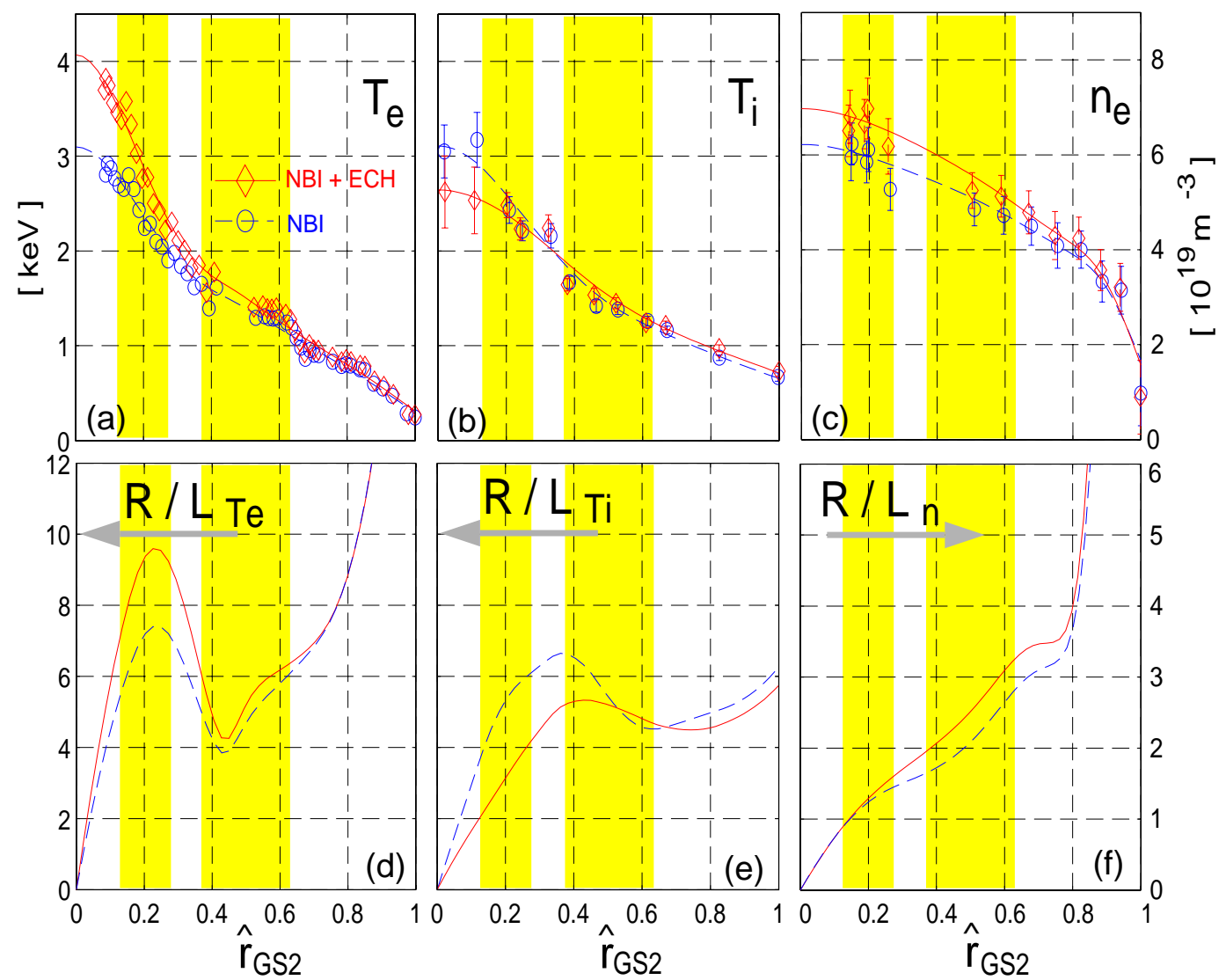

Figure 1 
Non-adiabatic passing electron response and outward impurity convection in gyrokinetic calculations of in
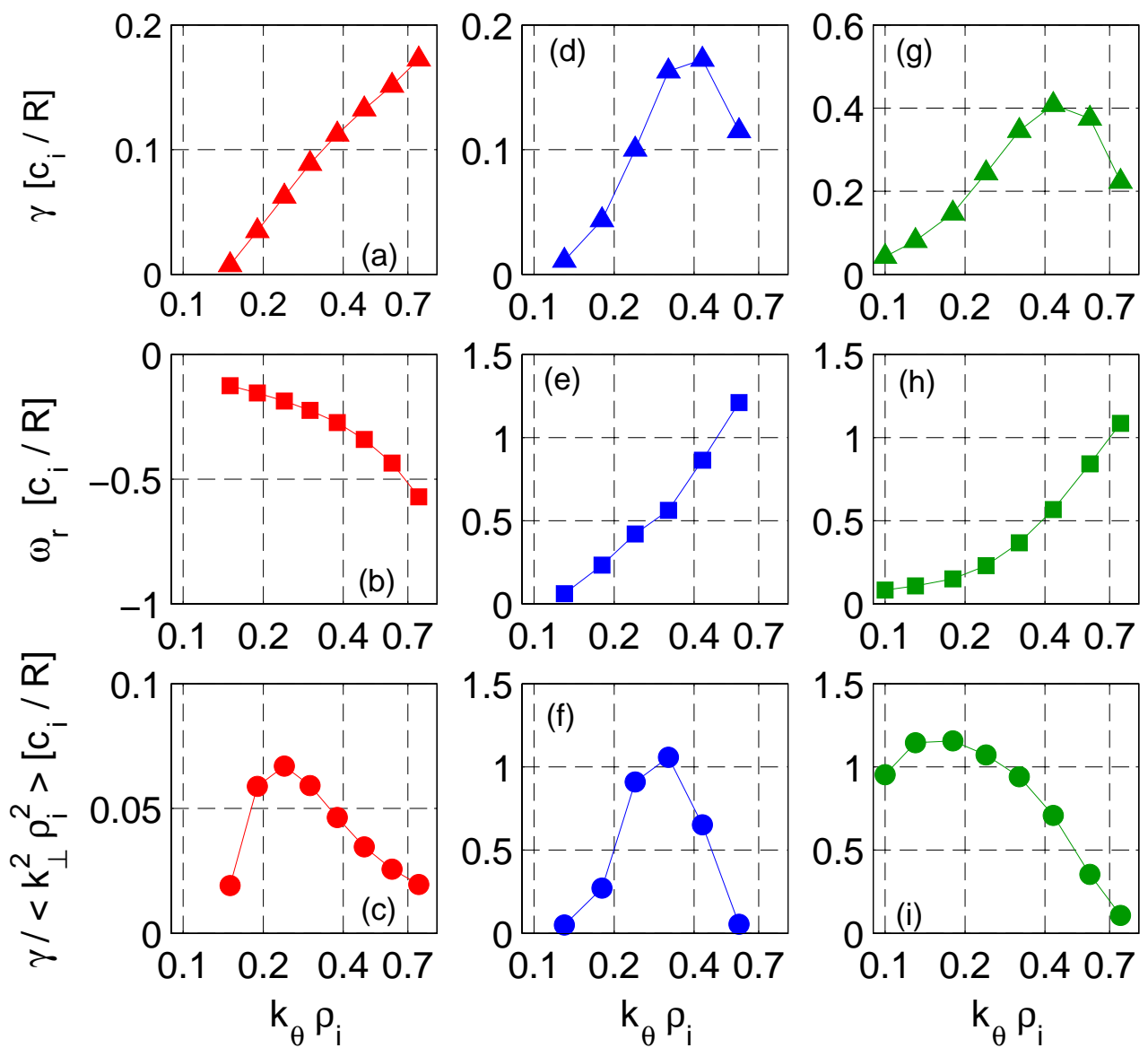

Figure 2 
Non-adiabatic passing electron response and outward impurity convection in gyrokinetic calculations of in

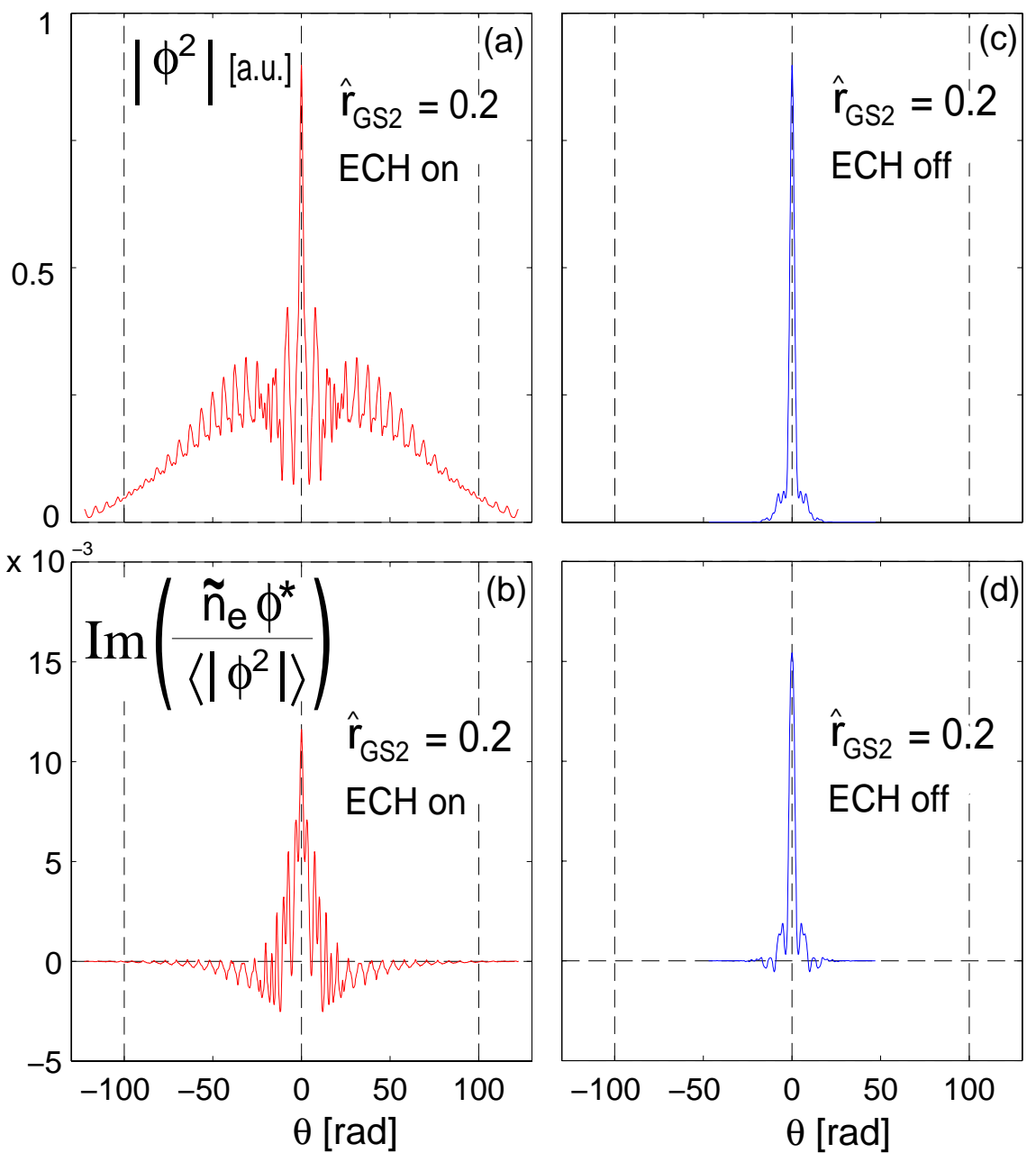

Figure 3

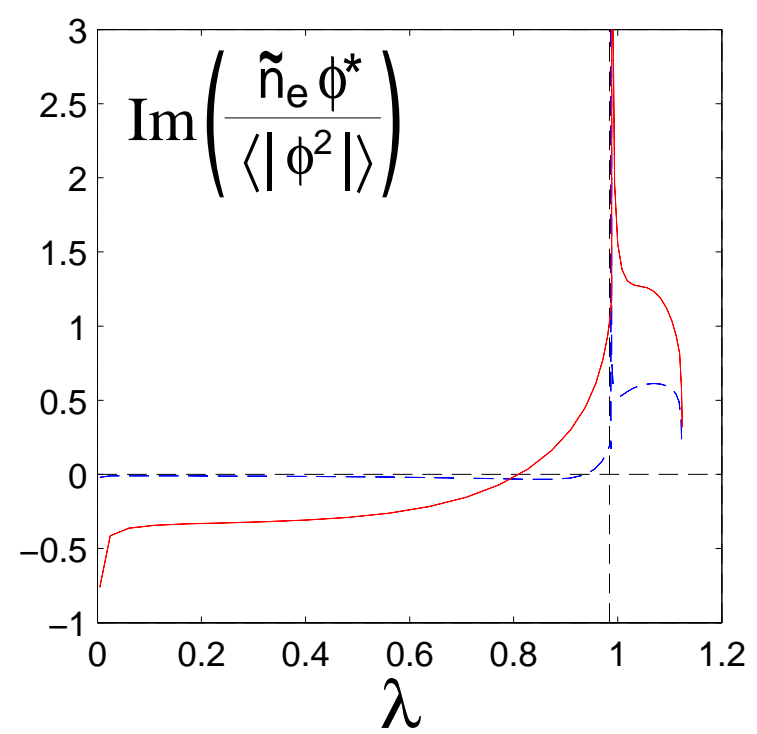

Figure 4 
Non-adiabatic passing electron response and outward impurity convection in gyrokinetic calculations of in
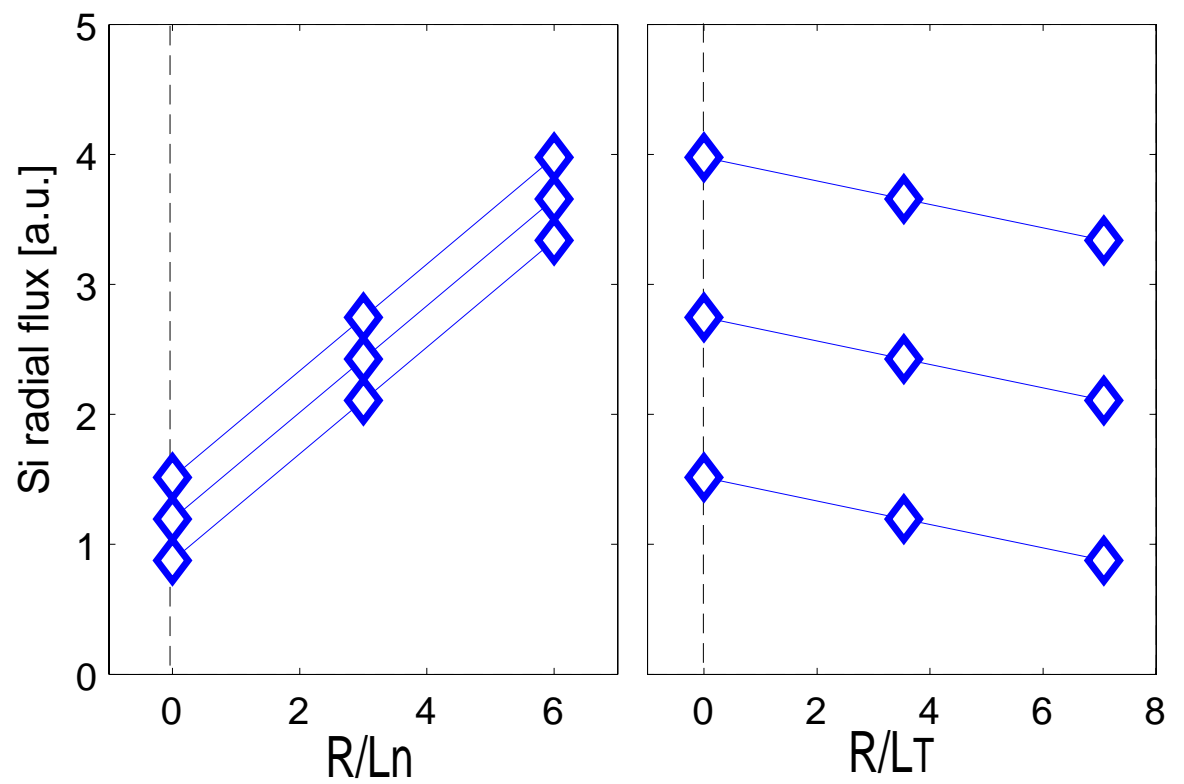

Figure 5
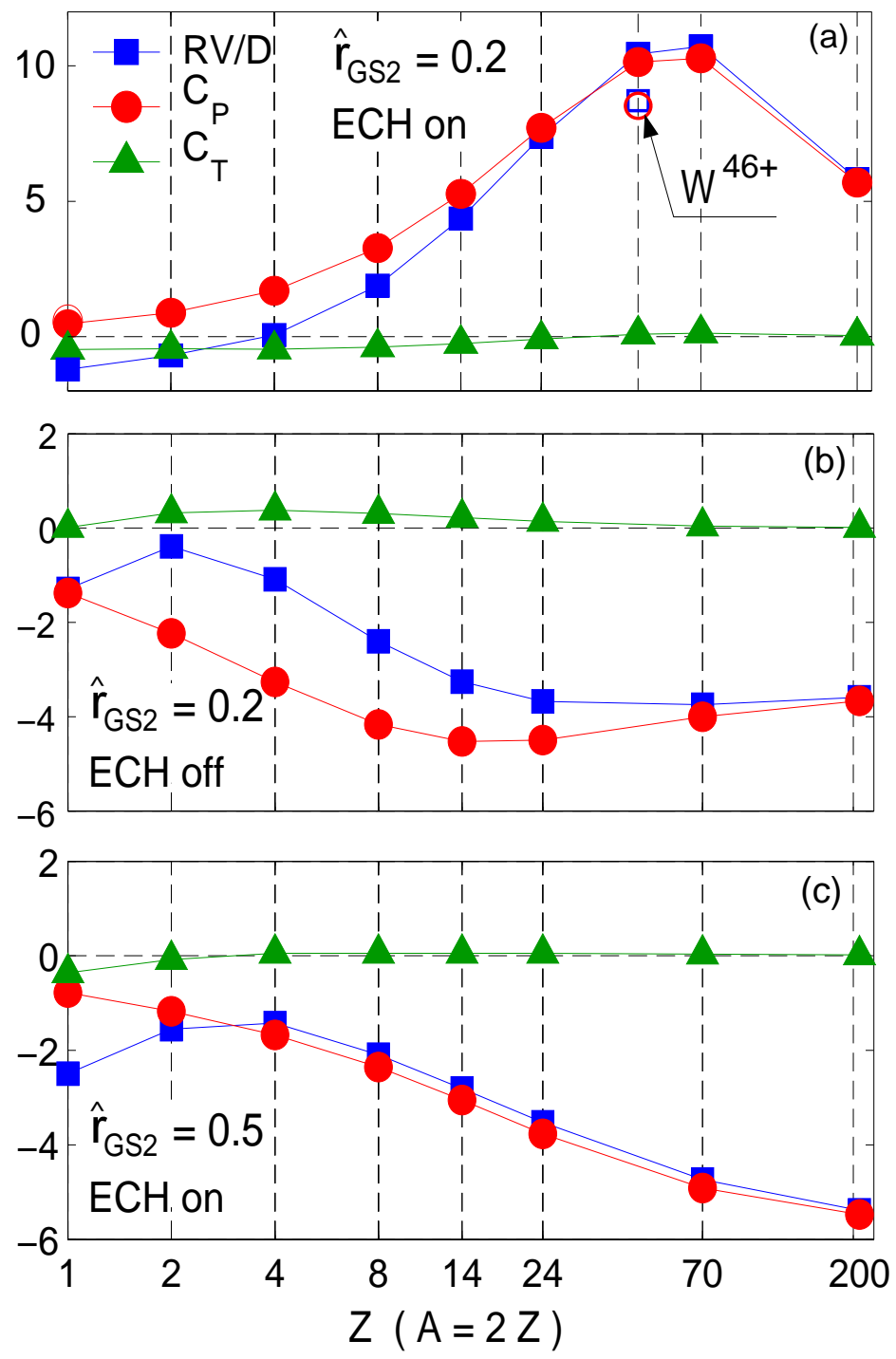
Non-adiabatic passing electron response and outward impurity convection in gyrokinetic calculations of in Figure 6

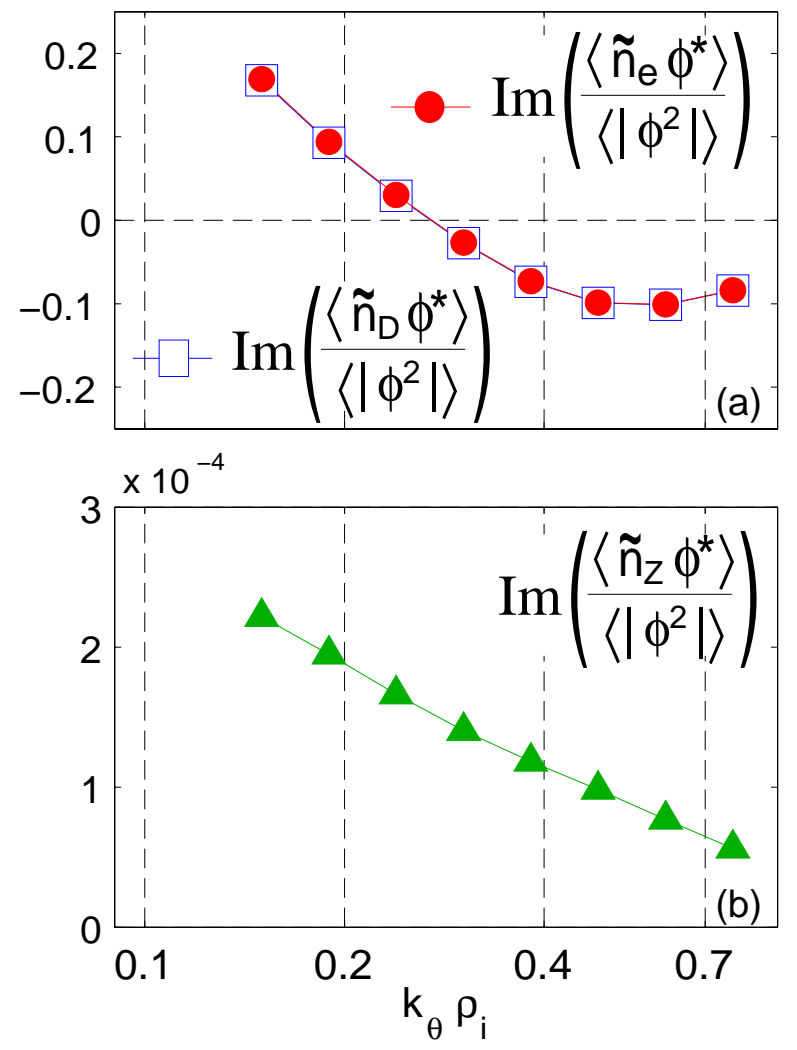

Figure 7 
Non-adiabatic passing electron response and outward impurity convection in gyrokinetic calculations of in

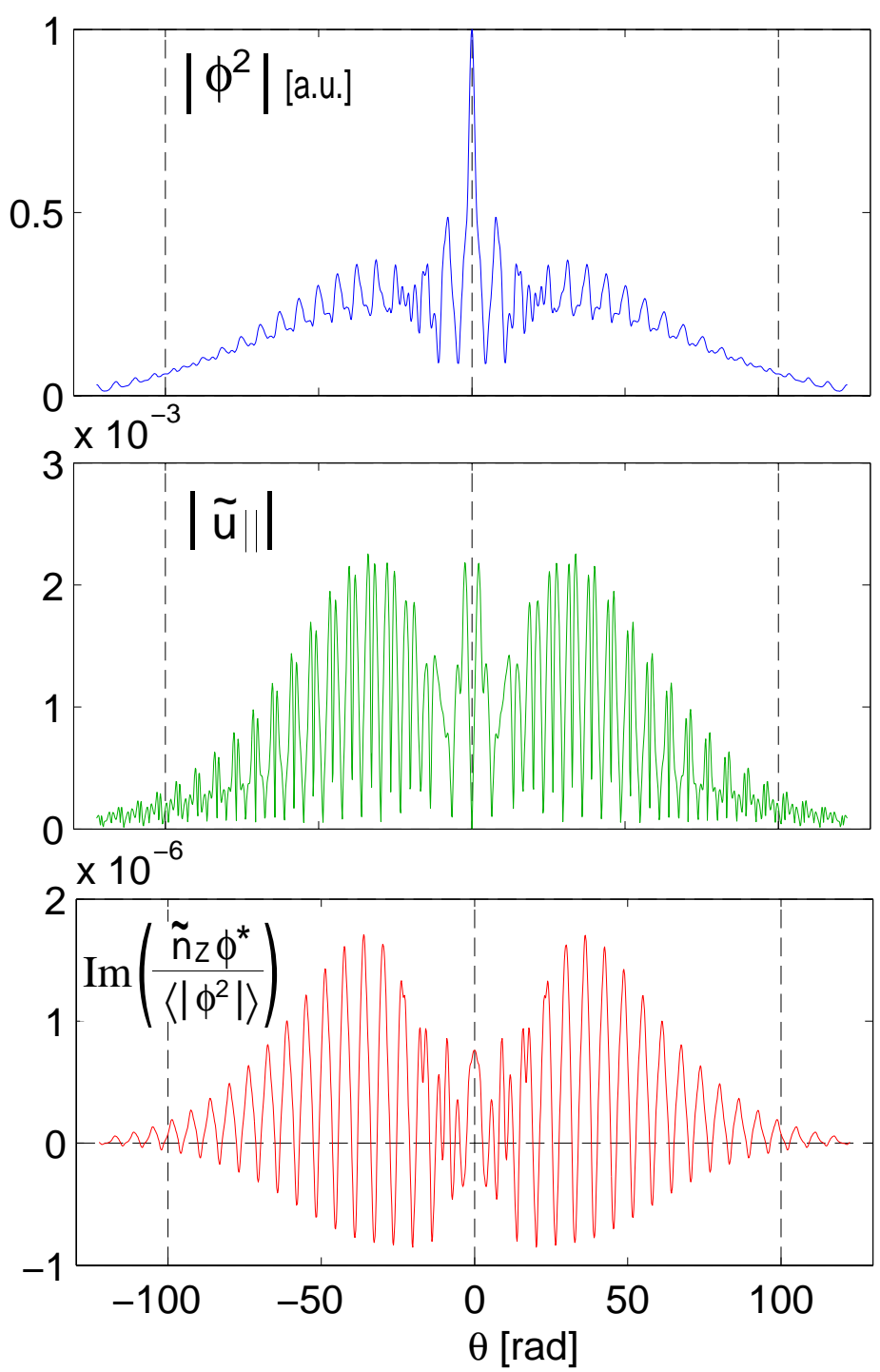

Figure 8 
Non-adiabatic passing electron response and outward impurity convection in gyrokinetic calculations of $i$
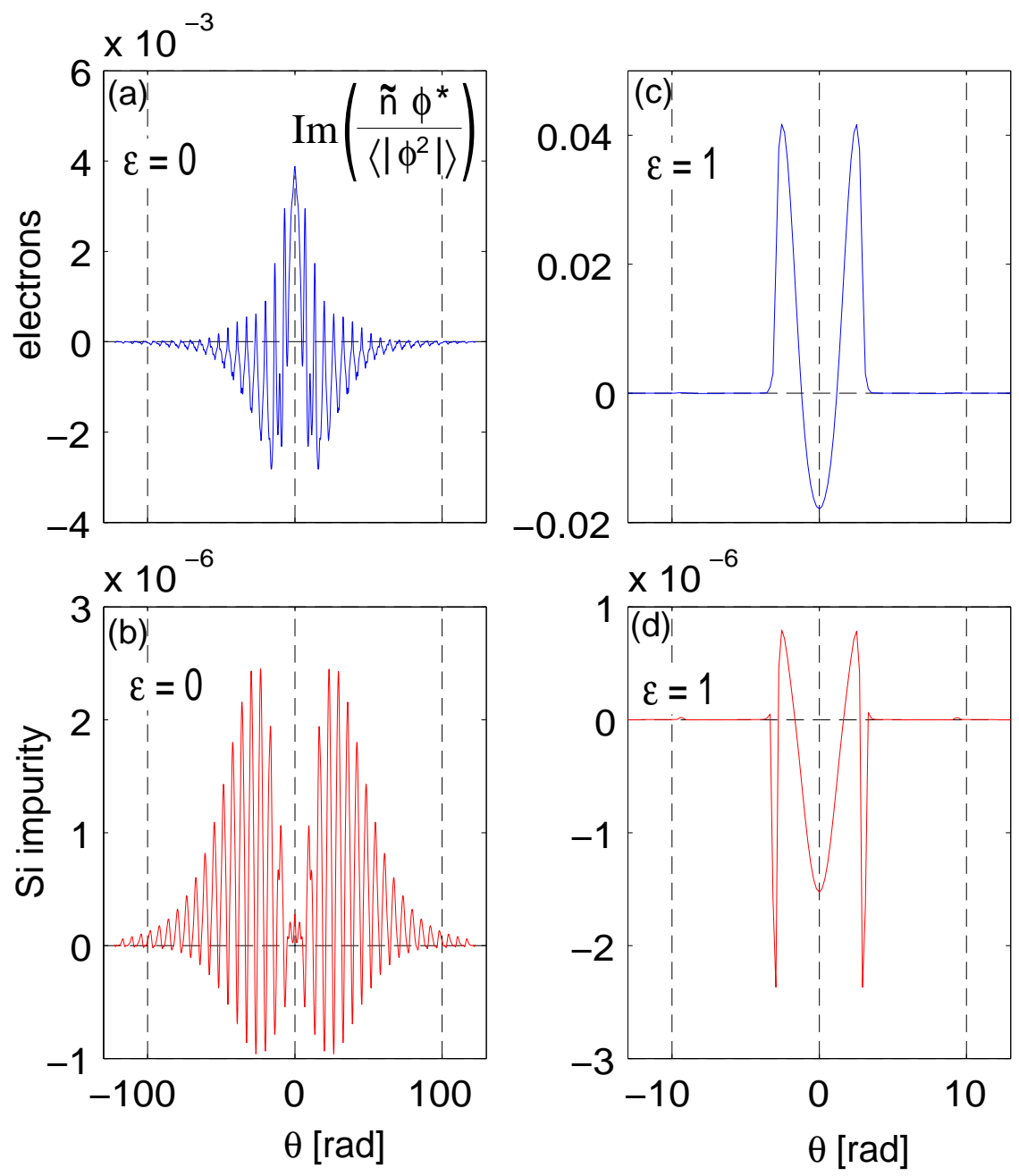

Figure 9 
Non-adiabatic passing electron response and outward impurity convection in gyrokinetic calculations of in
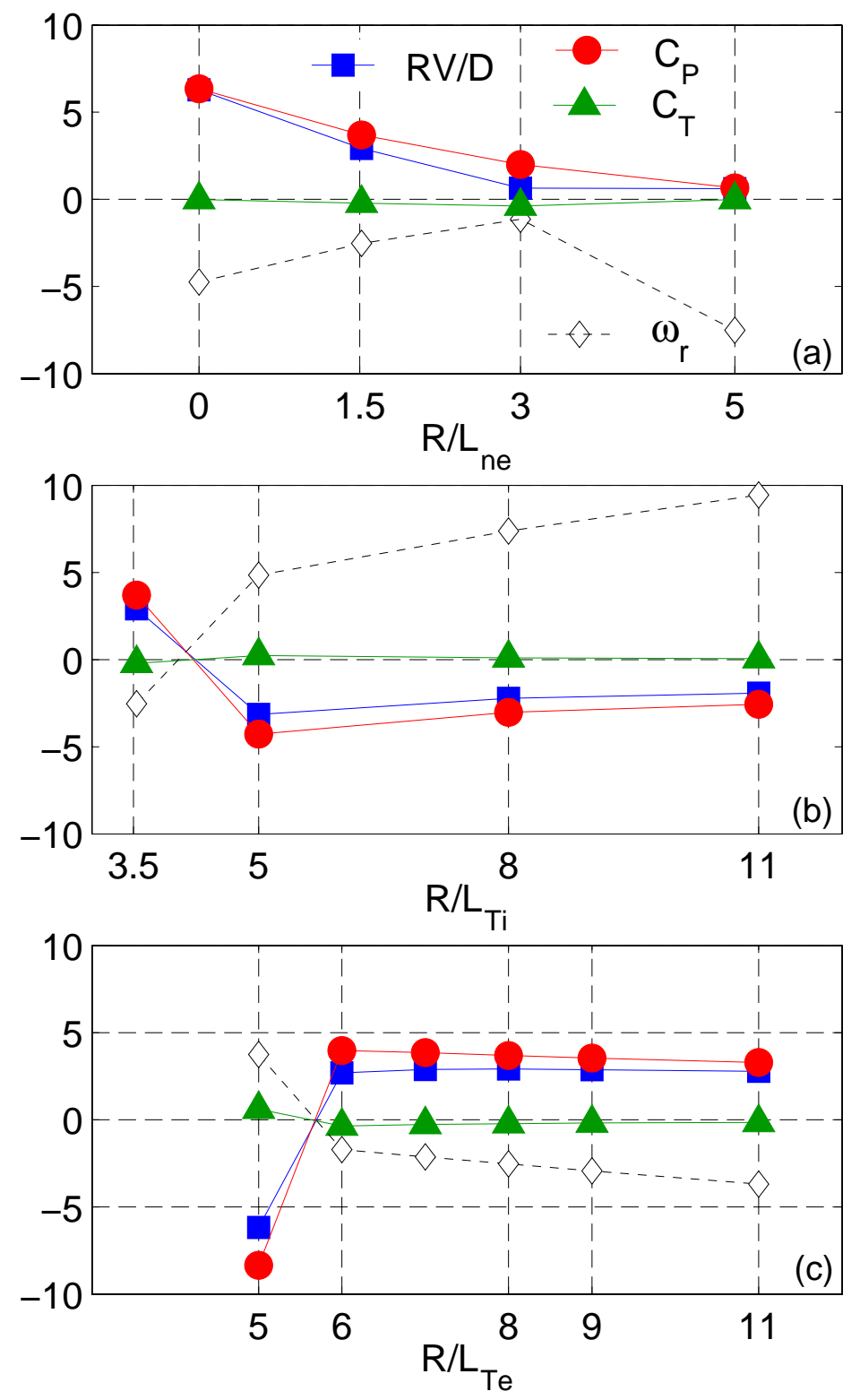

Figure 10 
Non-adiabatic passing electron response and outward impurity convection in gyrokinetic calculations of in
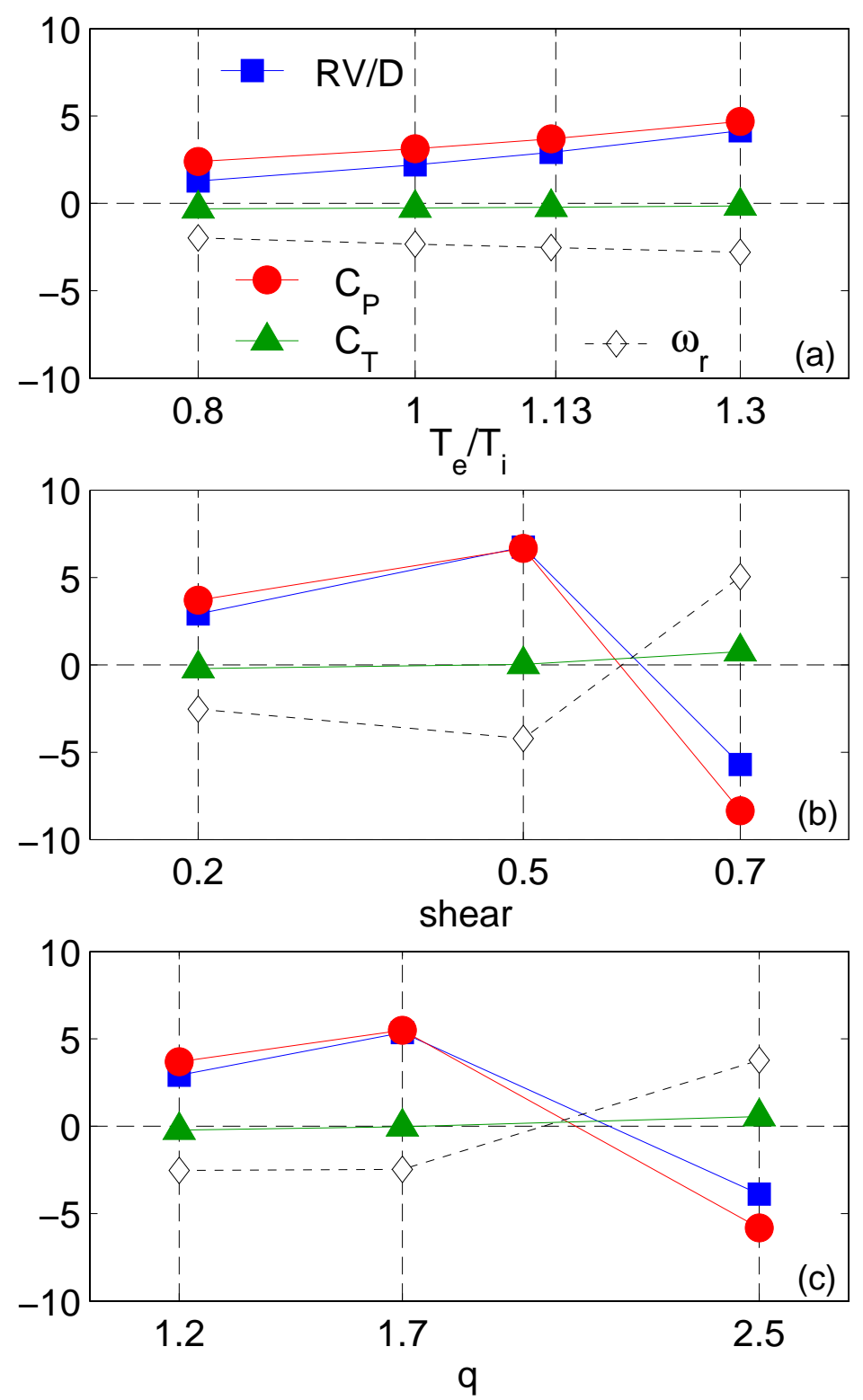

Figure 11 